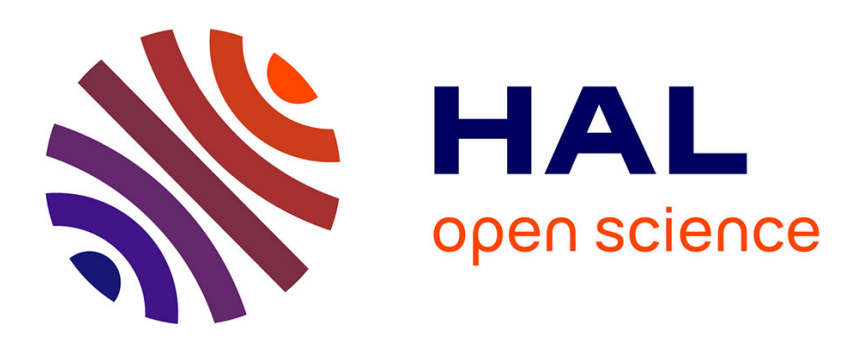

\title{
Transition to turbulence via spatiotemporal intermittency in one-dimensional Rayleigh-Bénard convection
}

\author{
F. Daviaud, M. Bonetti, M. Dubois
}

\section{- To cite this version:}

F. Daviaud, M. Bonetti, M. Dubois. Transition to turbulence via spatiotemporal intermittency in one-dimensional Rayleigh-Bénard convection. Physical Review A : Atomic, molecular, and optical physics [1990-2015], 1990, 42 (6), pp.3388 - 3399. 10.1103/PhysRevA.42.3388 . cea-01374027

HAL Id: cea-01374027

https://hal-cea.archives-ouvertes.fr/cea-01374027

Submitted on 29 Sep 2016

HAL is a multi-disciplinary open access archive for the deposit and dissemination of scientific research documents, whether they are published or not. The documents may come from teaching and research institutions in France or abroad, or from public or private research centers.
L'archive ouverte pluridisciplinaire $\mathbf{H A L}$, est destinée au dépôt et à la diffusion de documents scientifiques de niveau recherche, publiés ou non, émanant des établissements d'enseignement et de recherche français ou étrangers, des laboratoires publics ou privés. 


\title{
Transition to turbulence via spatiotemporal intermittency in one-dimensional Rayleigh-Bénard convection
}

\author{
F. Daviaud, M. Bonetti, and M. Dubois \\ Service de Physique du Solide et de Résonance Magnétique, Centre d'Etudes Nucléaires de Saclay, \\ F-91191 Gif-sur-Yvette CEDEX, France
}

(Received 7 March 1990)

\begin{abstract}
Rayleigh-Bénard convection is studied in quasi-one-dimensional geometries. Fixed and periodic boundary conditions are imposed using a rectangular and an annular cell, respectively. The destabilization process of the homogeneous convective pattern is studied for increasing Rayleigh number $\mathcal{R}$. The first time-dependent behaviors are given by the appearance of coupled oscillators. At larger $\mathcal{R}$ values, the spatial breakdown appears through the propagation of spatial defects, which appear to be solitary waves. This spatiotemporal destabilization is followed at higher $\mathcal{R}$ by a spatiotemporal intermittent regime, which corresponds to a dramatic decrease of the spatial coherence and to a mixing of turbulent patches within laminar domains. This last regime is studied within the frame of phase transitions. The statistical analysis evidences a second-order phase transition at least in the rectangular geometry (fixed boundary conditions), while this transition looks imperfect in the annular geometry (periodic boundary conditions). Nevertheless, the essential qualitative features shown by theoretical and numerical models are observed in both geometries. Comparison with a simple model of directed percolation shows that the imperfect nature of the transition in the annulus could be the consequence of some mechanism of self-generation of the turbulent domains. This mechanism is, however, unknown but is probably related to the influence of the boundaries.
\end{abstract}

\section{INTRODUCTION}

The evolution towards turbulent states of onedimensional (1D) hydrodynamical systems has recently received much interest. In fact, these systems are classified between the highly confined systems where deterministic chaotic dynamics might be observed and the extended systems in which developed turbulence generally takes place. Theoretical studies on these systems, which depend mainly on one space variable, have shown that a steady periodic cellular state may destabilize and become turbulent when a control parameter is varied. In this context, numerical simulations of phase equations, such as those derived from the Kuramoto-Sivashinsky equation, ${ }^{1-3}$ coupled map lattices, ${ }^{4,5}$ and cellular automata ${ }^{5}$ show that turbulence can be reached via spatiotemporal intermittency (STI). This STI regime corresponds to a mixture of organized and turbulent domains which exchange themselves in space and time.

In very recent years, experiments were done on 1D systems in which the basic state is spatially periodic. In this frame, Rayleigh-Bénard convection was studied in cells with narrow gaps (one horizontal dimension is much larger than the other) where only one space variable is involved. Specific spatial and dynamical properties were observed, as well as STI (Ref. 6) at high values of the Rayleigh number. Statistical analysis of STI regimes, performed under more adequate experimental conditions, ${ }^{7,8}$ has given results similar to those of numerical simulations. Nevertheless, the nature of the transition, which could be reminiscent of a direct percolation process, ${ }^{9}$ left some questions unanswered.
In order to get a deeper understanding of the dynamical regimes involved in this transition to turbulence-via STI - in 1D systems, we report detailed experimental results of convection in annular (periodic boundary conditions) and in rectangular (fixed boundary conditions) geometries. The two flows exhibit STI above a given Rayleigh number, but whereas the transition is almost perfect in the rectangular case, it turns out to be imperfect in the annulus, probably owing to the existence of local instabilities preceding the sustained STI regime.

In the following, we first describe the experimental setup and discuss the conditions relevant to the experiments (Sec. II). In Sec. III we present the regimes leading to STI and the existence of solitary waves. The statistical properties of STI in both geometries are discussed in Sec. IV. The use of a spatial criterion to discriminate laminar domains (LD's) from turbulent domains (TD's) allows us to perform a detailed statistical analysis in the case of the annular (Sec. IV A) and rectangular (Sec. IV B) geometries. In Sec. V we show numerical results of directed percolation and compare them with experimental data. Finally, in Sec. VI, the convective regimes in both geometries and the nature of the transition to STI are discussed.

\section{EXPERIMENTAL CONDITIONS}

Experiments were performed both in annular and rectangular cells filled with silicon oil of Prandtl number $\mathcal{P}=7$. Both geometries had vertical walls in Plexiglass. The annular and rectangular cells had sapphire and copper horizontal plates, respectively. In order to get a 
quasi-1D geometry, ${ }^{10}$ the annular cell had a circumferential aspect ratio $\Gamma_{c}=2 \pi r_{m} / d=35$ and a radial aspect ratio $\Gamma_{r}=\Delta r / d=0.29$ where $r_{m}, \Delta r$, and $d$ are the mean radius, the radial gap, and the cell depth $(d=7 \mathrm{~mm}$ for the two cells). In the rectangular cell the longitudinal aspect ratio was $\Gamma_{x}=L_{x} / d=25.7$ and the transverse aspect ratio was $\Gamma_{y}=L_{y} / d=0.43$. In the two sets of experiments the temperature difference across the fluid layer was kept constant to within $0.01 \mathrm{~K}$ by means of circulating thermal regulated water.

The convective structures were visualized by shadowgraphic imaging. A parallel light beam crossed vertically the annular cell through the horizontal sapphire plates, while in the rectangular cell, the beam was shone perpendicularly through the horizontal longest side $L_{x}$. In this way, the beam deflection was integrated along the cell depth (circular cell) and along the smallest horizontal dimension (rectangular cell). One had therefore the top view of the convective pattern in the circular geometry and the front view in the rectangular one (see Fig. 1).

The temporal evolution of the annular roll pattern was recorded using either a circumferentially moving photodiode positioned at the place of the shadowgraphic image or a video camera recording system. With the former technique, the annular convective structure was azimuthally scanned every second over 260 spatial points giving near the onset of STI a spatial resolution of $\sim 6$ points/wavelength. The video technique was used in both geometries and, as the convective dynamics is quasi-1D, the shadowgraphic image was digitized along a circle of approximately 1200 pixels (annular cell) or an horizontal line of 512 pixels (rectangular cell), giving a spatial resolution of $\sim 30$ pixels and $\sim 16$ pixels per wavelength, respectively. The circle and line were appropriately selected in order to get a representative skeleton of the convective pattern, without losing too much information on the flow dynamics. The acquisition frequency could be increased up to 5 circles/sec and 20 lines/sec for the annulus and rectangle, respectively.

In both geometries the time lapse between each new line acquisition was set according to which dynamics, i.e., a short or a long time evolution, was studied. For example, when we analyzed the spatiotemporal intermittency, we looked particularly at the long time evolution of the roll pattern. In the rectangular cell, up to 1900 lines were recorded with a time interval generally set to $3 \mathrm{sec}$; in the annular cell, time series up to $2000 \mathrm{sec}$ were recorded.
The total acquisition time is to be compared to a characteristic time, namely, the basic period $T_{0}$ of the rolls' oscillators. As $T_{0} \simeq 1$ and $2 \mathrm{sec}$ in the rectangular and annular cells, respectively, this yields a total acquisition time of about 6000 and 1000 basic oscillations of the rolls.

After each temperature increase, dynamical equilibrium must be reached. In both experiments, we waited as long as $24 \mathrm{~h}$ up to $48 \mathrm{~h}$ before each new data acquisition. As a matter of fact, these delays revealed themselves sufficient to obtain a new equilibrium dynamical regime. The comparison with the phase diffusion time $\tau_{D}$ $=L_{x}^{2} / D_{\|}$cannot easily be done since $D_{\|}$is not known for these narrow geometries and high Rayleigh numbers. Nevertheless, taking $D_{\|}=2.2 \times 10^{-3} \mathrm{~cm}^{2} \mathrm{sec}^{-1}$ as calculated (and measured) near the convective onset, ${ }^{11}$ these delays are considered sufficient $\left(\tau_{D} \simeq 2\right.$ and 3 days for the rectangular and the annular geometries, respectively).

\section{FLOW EVOLUTION BELOW THE SPATIOTEMPORAL INTERMITTENT REGIME}

Spatial and dynamical properties specific to the narrow channels, (i.e., $\Gamma_{y}<0.5$ ), have been already reported elsewhere. ${ }^{12}$ They are summarized as follows. When $\mathcal{R}$ exceeds a critical value $\mathcal{R}_{c}=f\left(\Gamma_{y}\right)$, perfect stationary patterns are observed with the roll's axis perpendicular to the longest side of the container. An increase of $\mathcal{R}$ induces generally the appearance of new rolls, making the wavelength $\lambda$ of the pattern much smaller than the one observed in large containers. ${ }^{13}$ Wavelengths down to $2 L_{y}$ can be obtained. The value of $\lambda$ depends on the Rayleigh number and on the thermal history. Near the onset of STI and in a reproducible manner, $30\left(\lambda_{0}=0.43 \lambda_{c}\right.$ with $\left.\lambda_{c}=2 d\right)$ and $40\left(\lambda_{0}=0.44 \lambda_{c}\right)$ wavelengths $\lambda_{0}$ are present in the rectangular channel and the annular channel, respectively. In the annular geometry, at lower $\mathcal{R}$ values, a stable spatial inhomogeneity of the local wavelength is generally observed and up to now, no clear explanation of this phenomenon has been given.

Above a given $\epsilon$ value $\left[\epsilon=\mathscr{R} / \mathcal{R}_{c}\left(\Gamma_{y}\right)-1\right]$ which depends on the transverse aspect ratio $\Gamma_{y}$ and on the present wavelength, the pattern becomes time dependent with the appearance of thermal oscillators (domain labeled by $D$ in Fig. 2).

In the rectangular channel, at $\epsilon=310$, hot plumes ap-

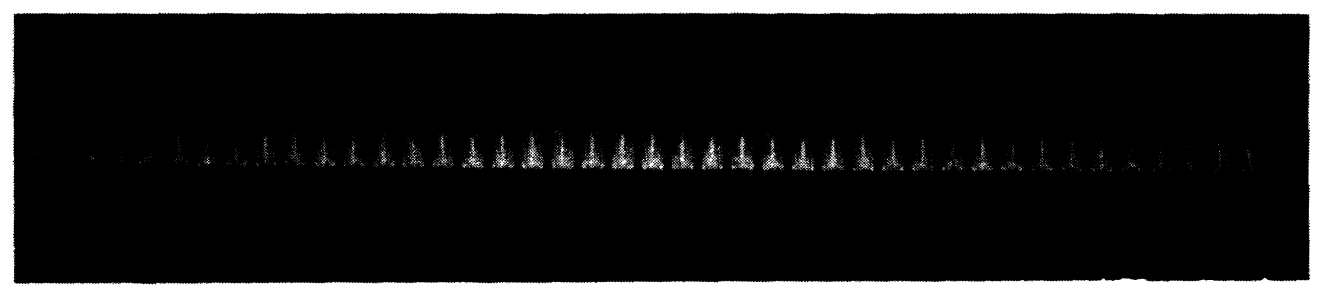

FIG. 1. Shadowgraphic image of 1D Rayleigh-Bénard convection in a rectangular container with $\Gamma_{y}=0.3$ at $\epsilon=45$. Bright and dark lines are cold and warm currents, respectively. 
(a)

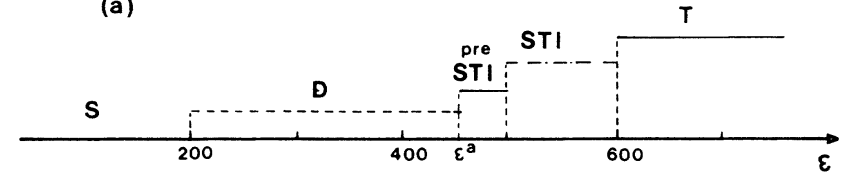

(b)

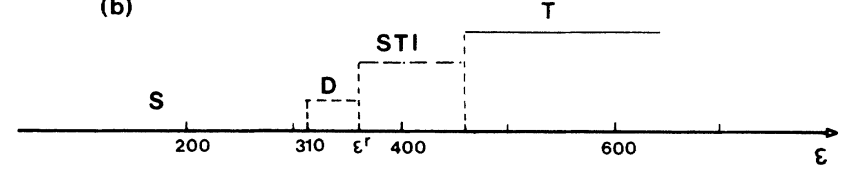

FIG. 2. Phase diagram of the convective state as a function of $\epsilon$ in the annular cell (a) and in the rectangular channel (b). S stands for a stationary pattern, D for a time and/or spatial oscillating pattern, STI for spatiotemporal intermittency, and T for a complete disorganized and turbulent pattern.

pear periodically in a pattern with a wavelength $\lambda=0.45 \lambda_{c}$. The system is at first monoperiodic, i.e., all the plumes have the same frequency everywhere and the convective rolls are similar to a chain of coupled oscillators.

In the annular cell, collective oscillations are observed for $\epsilon \geq 200$ and are related to a mechanism of vacillations, as it has been described in Ref. 12. We recall that these vacillations, which correspond to a periodic displacement, in phase opposition, of the ascending and descending streams around their mean position, are specific to the narrow channels with $\Gamma_{y} \leq 0.40$. When the Rayleigh number is further increased, the collective periodic behavior may become chaotic with properties similar to those of a pure dynamical system, while the spatial properties remain unchanged. Still in the annulus, a small evolution of the pattern, probably induced by a slow and a nonhomogeneous drift of the phase of the rolls can also be observed, but globally the spatial features of the pattern remain unchanged with a wavelength dispersion as already mentioned.

At a larger value of $\epsilon$, new events leading to a spatial symmetry breaking appear. These events were observed in a stationary pattern present in a rectangular channel between horizontal glass plates. ${ }^{10}$ The behavior is the following: in an otherwise perfect pattern, a local destabilization produces a wavelength larger than its neighbors. This defect, which extends over two or three wavelengths, propagates along the pattern and can be reflected at the lateral walls while keeping its topological aspect (Fig. 3). Therefore it looks like a solitary wave. These waves, which have been further observed within the experimental conditions of the results reported here, are difficult to detect because their velocity is very low $\left[\left(10^{-2}-5 \times 10^{-2}\right) \lambda_{0} \mathrm{sec}^{-1}\right.$ with $\lambda_{0}$ the basic wavelength of the pattern] and their dynamic is combined with the behaviors of the oscillators. In the studied rectangular channel where hot plumes are present, the defect propagates only in part of the layer and can be reflected by organized domains that are very robust, at least for a given time [Fig. 4(a)].

In the annular channel, these solitary waves have also

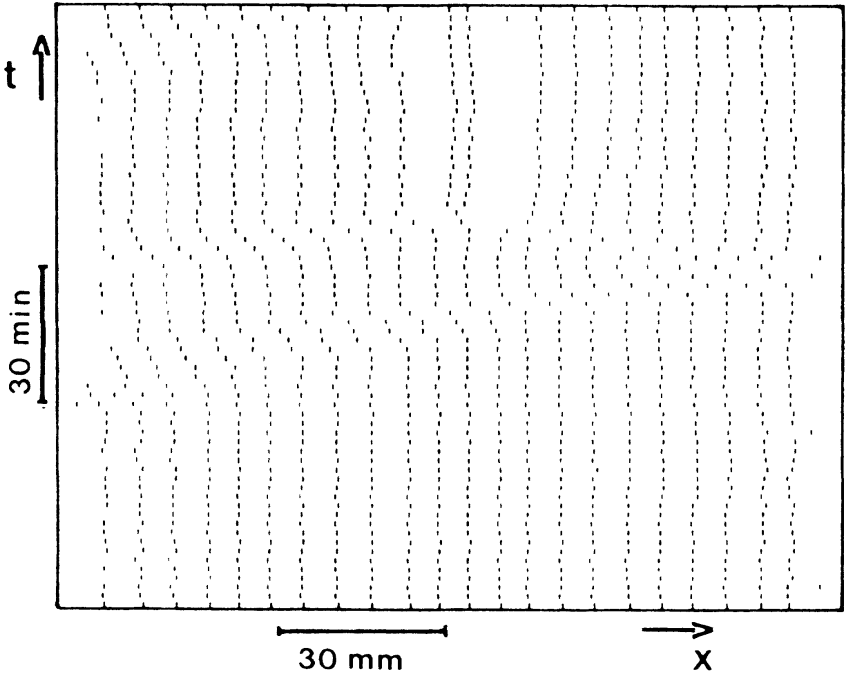

FIG. 3. Time evolution of the shadowgraphic minima in a rectangular cell (between horizontal glass plates) with $\Gamma_{x} \simeq 21$ and $\Gamma_{y} \simeq 0.35$. Notice the collapse of two solitary waves which gives rise to two local large cells.

been observed just below the threshold of STI. In fact, these waves appear in an $\epsilon$ domain which corresponds also to a homogeneity of the local wavelengths that are no longer dispersed at the threshold of STI. There could be a relation between the two phenomena though it has not been evidenced. Figure 5(a) shows the existence of such waves in different directions. It is difficult to know whether they propagate all around the cell and cross each other or are reflected by domains of oscillating cells.

In all the experiments, these waves are observed just below the onset of local turbulent events and it seems likely that they have a fundamental role in their appearance. Indeed, when two defects propagating in opposite directions collapse, they give rise to large local cells that are origins for turbulent patches (Fig. 3). Their interaction with oscillators can also destabilize locally the pattern. The convective pattern enters then in the regime of spatiotemporal intermittencies.

\section{SPATIOTEMPORAL INTERMITTENCY}

In both geometries, above a given $\epsilon$ value, the pattern shows a mixture of organized laminar domains and incoherent turbulent domains (later defined) that are fluctuating in space and time. The first events of intermittency are observed for $\epsilon^{a}=450$ in the annular cell and $\epsilon^{r}=350$ in the rectangular channel (Fig. 2). Here, superscripts $a$ and $r$ will refer, respectively, to the annular and rectangular geometries.

Annular channel. In the range $\epsilon^{a}<\epsilon<500$, the dynamical regime in the annulus is characterized by turbulent patches which are localized both in space and time. These TD's can reach a spatial extension up to ten rolls with a lifetime shorter than $100 T_{0}$, with $T_{0}$ the typical time period of the roll's oscillation. They can propa- 


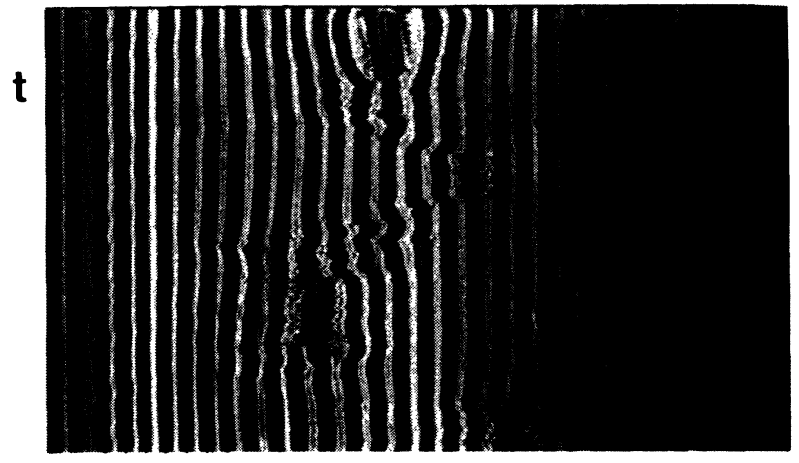

(a)

$\mathbf{x}$

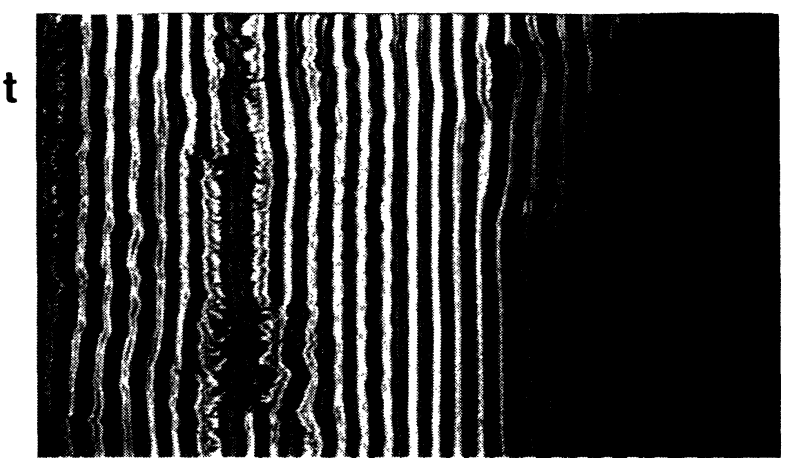

(b)

$\mathbf{x}$

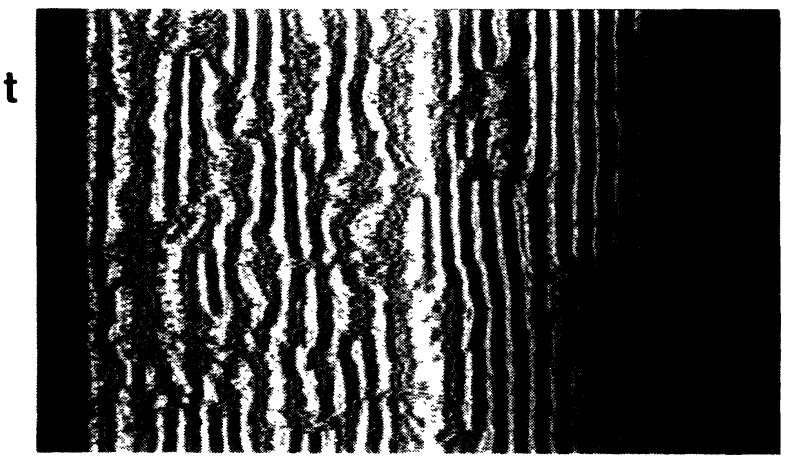

(c)

$\mathbf{x}$

FIG. 4. Space-time evolution of the convective pattern obtained in the rectangular channel from the shadowgraphic images. The light intensity is plotted using a gray scale with 256 levels. Spatial digitization is made over 512 pixels (horizontal axis). The total observation time (vertical axis) is $1536 \mathrm{sec}$. Vertical dark lines correspond to stationary warm ascending currents. (a) $\epsilon=350$, (b) $\epsilon=409$, (c) $\epsilon=561$.

gate around the container by contamination but they do not spread. Moreover, they can be separated by very long periods of complete ordered regimes (up to several hours near $\epsilon^{a}$ ). When $\epsilon$ is increased, the spatial and temporal extension of these domains increases while the periods of ordered regimes become more and more seldom. Around $\epsilon=500$, a fluctuating regime made of LD's and TD's is always present in all the container and is
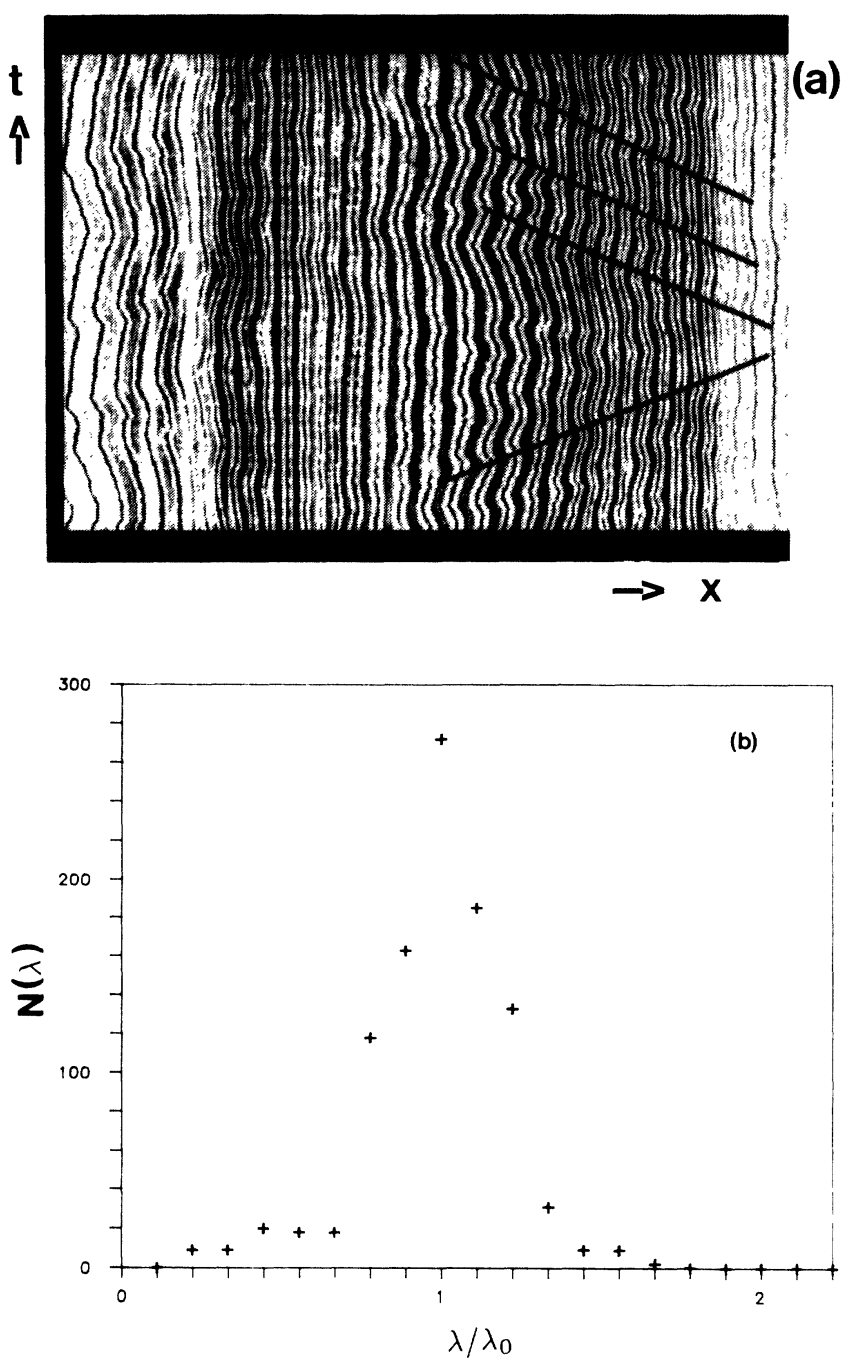

FIG. 5. (a) Same as in Fig. 4 but with the annular channel. The total observation time is $1500 \mathrm{sec}$ and $\epsilon=420$. Notice at the right and left of the frame the crossing of solitary waves. Dark lines indicate the trajectory of some solitary waves. (b) Wavelength distribution at $\epsilon=420$.

characteristic of sustained intermittency. The propagation of the TD's through the pattern of LD's has a treelike structure (Fig. 6) as is observed in the spatiotemporal diagrams of directed percolation (see Sec. V).

Rectangular channel. The transition in the rectangular cell from the organized "laminar" state to the STI can be observed in Fig. 4. The behavior looks qualitatively the same as in the annular cell, at least near the threshold, with the coexistence of laminar and turbulent domains, however with a stronger spatial confinement of the turbulent regions. As can be seen in Fig. 4(b), turbulent patches are sandwiched between laminar domains and hardly propagate throughout the cell.

The simultaneous coexistence of two kinds of qualitatively different domains implies the search for a criterion which distinguishes their different spatial and temporal characteristics. LD's are regions where the initial spatial 


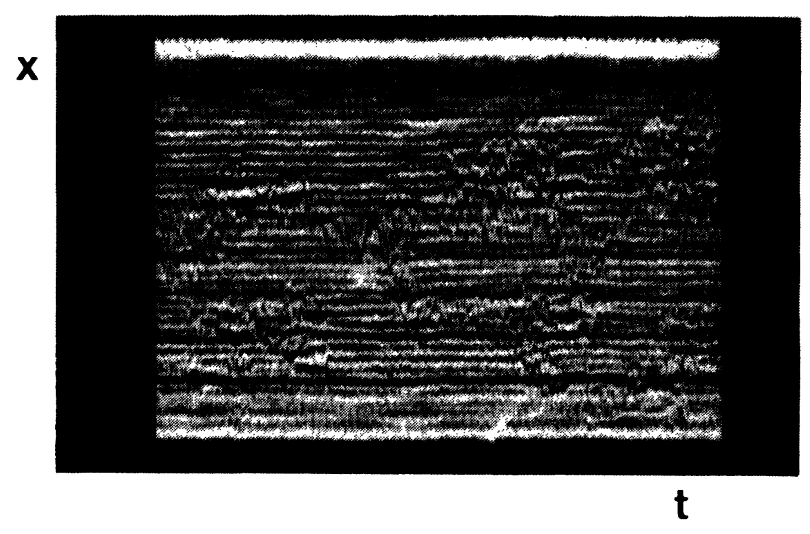

FIG. 6. Same as in Fig. 4 but with the annular convective pattern at $\epsilon=520$. The total observation time is $1000 \mathrm{sec}$ (along the horizontal axis, time is going from left to right) and the spatial digitization is made over 250 pixels. Notice the contamination process of the turbulent domains throughout the laminar ones.

periodicity of the roll structure is preserved, see Fig. 4(b). Furthermore, in LD's the time evolution of the shadowgraphic intensity $I(x, t)$ at a fixed given point is either stationary or oscillating in time leading to a local monoperiodic or quasiperiodic regime with a small noise level. We recall that the oscillations correspond to the vacillations in the annulus and to ascending plumes in the rectangular cell. On the contrary, TD's are regions without spatial coherence (see Sec. IV B) and their time evolution is chaotic. This difference between LD's and TD's is well evidenced in Fig. 4(c).

The choice of a spatial criterion based on the local wavelength is therefore justified to discriminate LD's from TD's without ambiguity. ${ }^{8}$ A reduction of the signal can thus be performed, first by finding the extrema of the intensity $I(x, t)$ (a maximum corresponding to a cold stream and a minimum to a hot one). A region in the convective pattern between two consecutive extrema $x_{i}$ and $x_{t+1}$ of $I$ is then called laminar if

$$
\lambda_{0}\left(\frac{1}{2}-\Delta\right)<x_{i+1}-x_{i}<\lambda_{0}\left(\frac{1}{2}+\Delta\right)
$$

$\Delta$ being a tolerance factor and $\lambda_{0}$ the mean wavelength, otherwise it is called turbulent. We have checked that the result does not depend on $\Delta$ provided $\frac{1}{8} \leq \Delta \leq \frac{1}{4}$. Such a choice is suggested by the peaked distribution of the wavelengths as shown in Fig. 5(b). The signal can then be reduced to a binary representation of $I$ (laminar or turbulent) which allows us to perform statistics on LD's and TD's. We have also used a temporal criterion to validate the previous data reductions. Intensity differences between successive acquisitions were computed and compared to a cutoff value $\delta$. A pixel $i$ is called laminar if

$$
I(i, t+1)-I(i, t) \leq \delta,
$$

otherwise it is called turbulent. The results are similar, but with a larger dispersion, to those obtained with the spatial criterion. In the following, we present results
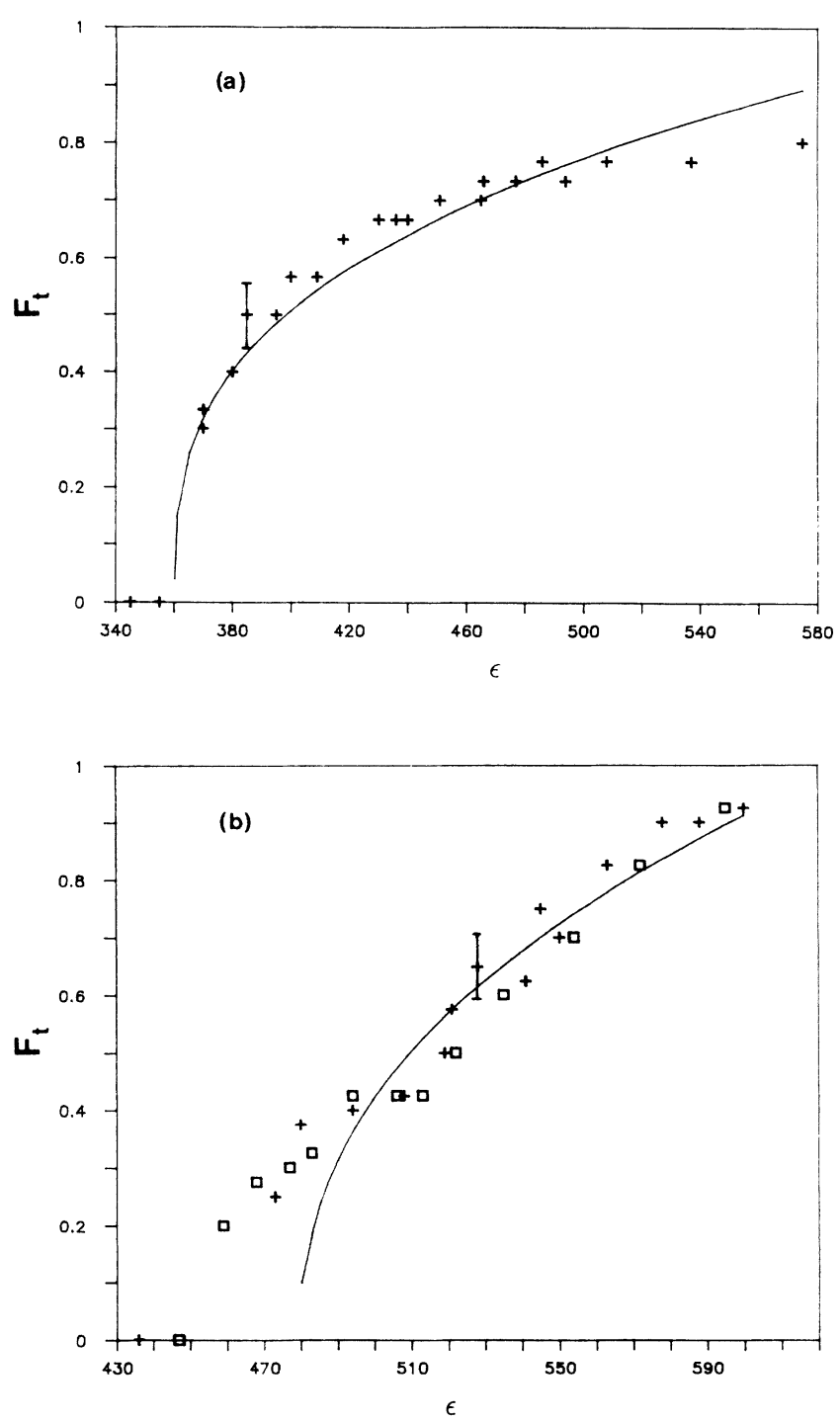

FIG. 7. Mean turbulent fraction $F_{t}$ as a function of $\epsilon$. (a) Rectangular channel, (b) annular cell. See text for the full curves.

which have been computed using the spatial criterion.

In order to characterize the global degree of chaos, we have computed the mean turbulent fraction $F_{t}: F_{t}$ refers to the averaged total length occupied by the turbulent cells divided by the total length of the container. $F_{t}$ is plotted as a function of $\epsilon$ for the rectangular [Fig. 7(a)] and the annular [Fig. 7(b)] geometry. The difference between the two geometries is striking even if they both show a continuous increase of $F_{t}$ with $\epsilon$. In the rectangular case, the transition looks like a quasiperfect phase transition and a fit of the experimental data gives the relation

$$
F_{t} \sim\left(\epsilon-\epsilon_{F}^{r}\right)^{\beta^{r}}
$$

with $\epsilon_{F}^{r}=360$ and $\beta^{r}=0.3 \pm 0.05$. In the annular geometry, the transition to STI appears not well defined. 
Notice that in both geometries, no hysteretic phenomenon has been observed when increasing or decreasing the Rayleigh number.

In order to get more information on the nature of the transition and to understand the difference observed in the two geometries, a detailed statistical analysis has been made. We present the results separately for each case in the following.

\section{A. STI in annular geometry}

The distributions of spatial lengths of LD's have been computed. Figure 8 shows the histograms of the number $N(L)$ of LD's with length $L$ for different values of $\epsilon$. One must notice that the length $L$ is computed in term of laminar cells, so $L$ can take values between 1 and 80 in the annular geometry. When $\epsilon$ is varied, two kinds of distributions are evidenced. In the range $460<\epsilon<500$, the

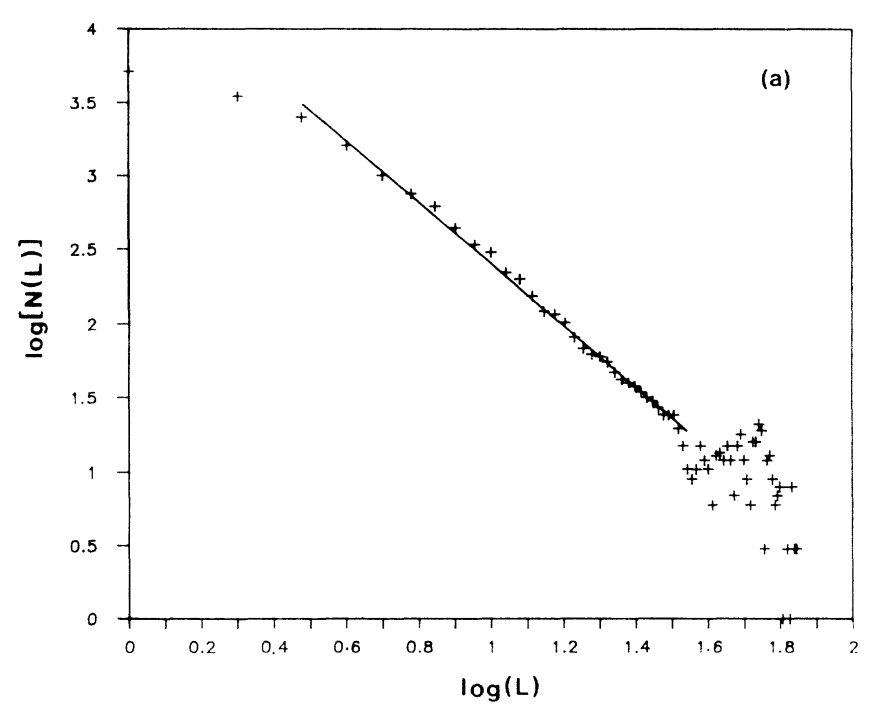

histogram shows a power-law decay, in a domain of lengths $L$ which is $\epsilon$ dependent.

The power-law decay which can be estimated on the largest available range of scales at $\epsilon=\epsilon_{s}^{a}=480 \pm 5$ has a characteristic exponent $\mu_{s}^{a}=1.7 \pm 0.1$ [Fig. 8(a)]. According to a theoretical definition, ${ }^{14}$ this value $\epsilon_{s}^{a}$ could be defined as the threshold of STI. If we plot the relation $F_{t} \sim\left(\epsilon-\epsilon_{s}^{a}\right)^{\beta}$ with $\beta=\beta^{r}=0.3$ as computed in the rectangular channel, we can fit the experimental points only at the large values of $\epsilon$. The discrepancy observed at the low values of $\epsilon$ could be related to an imperfect nature of the transition as will be discussed in Sec. V.

For $\epsilon>\epsilon_{s}^{a}$ the histogram can be divided into two parts: a first part in the range of the small scales shows an algebraic decay with the same exponent $\mu_{s}^{a}$ while at large scales an exponential decay is evidenced [Fig. 8(b)]. The histograms exhibit this crossover phenomenon until $\epsilon=500$, from where they are best fitted by an exponential

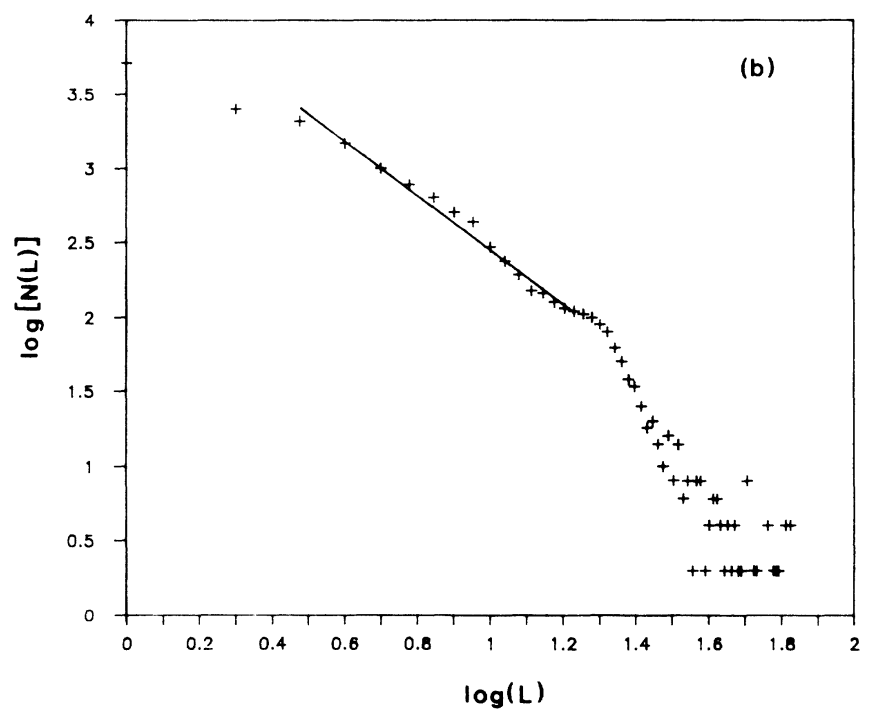

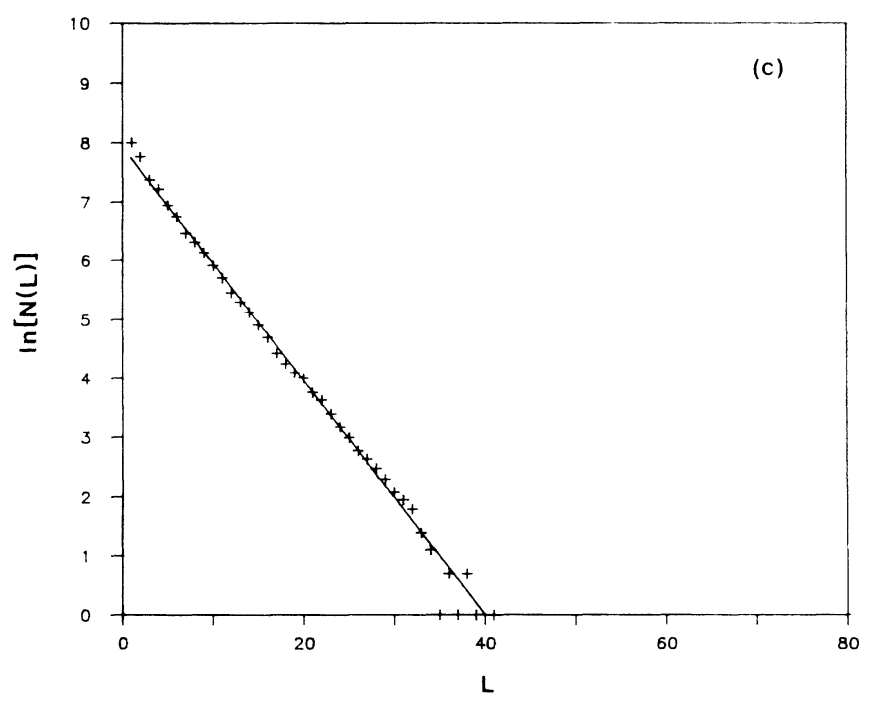

FIG. 8. Histograms $N(L)$ of laminar domains of length $L$. (a) Power-law distribution at $\epsilon=\epsilon_{s}^{a}=480$, (b) $\epsilon=500$, (c) exponential distribution at $\epsilon=530$. 
over the whole range of lengths. Figure $8(\mathrm{c})$ shows this exponential decay for $\epsilon=530$. If $m_{s}$ is the slope of the curve $\ln [N(L)]$ versus $L$, the characteristic length $l_{s}=1 / m_{s}$ is observed to decrease when $\epsilon$ increases. A plot of $m_{s}^{2}$ as a function of $\epsilon$ [Fig. 9(a)] reveals two kinds of behavior: at the lowest values of $\epsilon$ for which $m_{s}$ can be computed, $m_{s}^{2}$ has a nearly constant value, while at larger values of $\epsilon, m_{s}^{2}$ increases almost linearly as a function of $\epsilon$. This linear behavior is similar to the one observed in the rectangular channel and in the experiment of Ciliberto and Bigazzi. ${ }^{7}$ However, the global behavior remains complex and if there exists a transition-in the sense of phase transition - these experimental results evidence its imperfect nature.
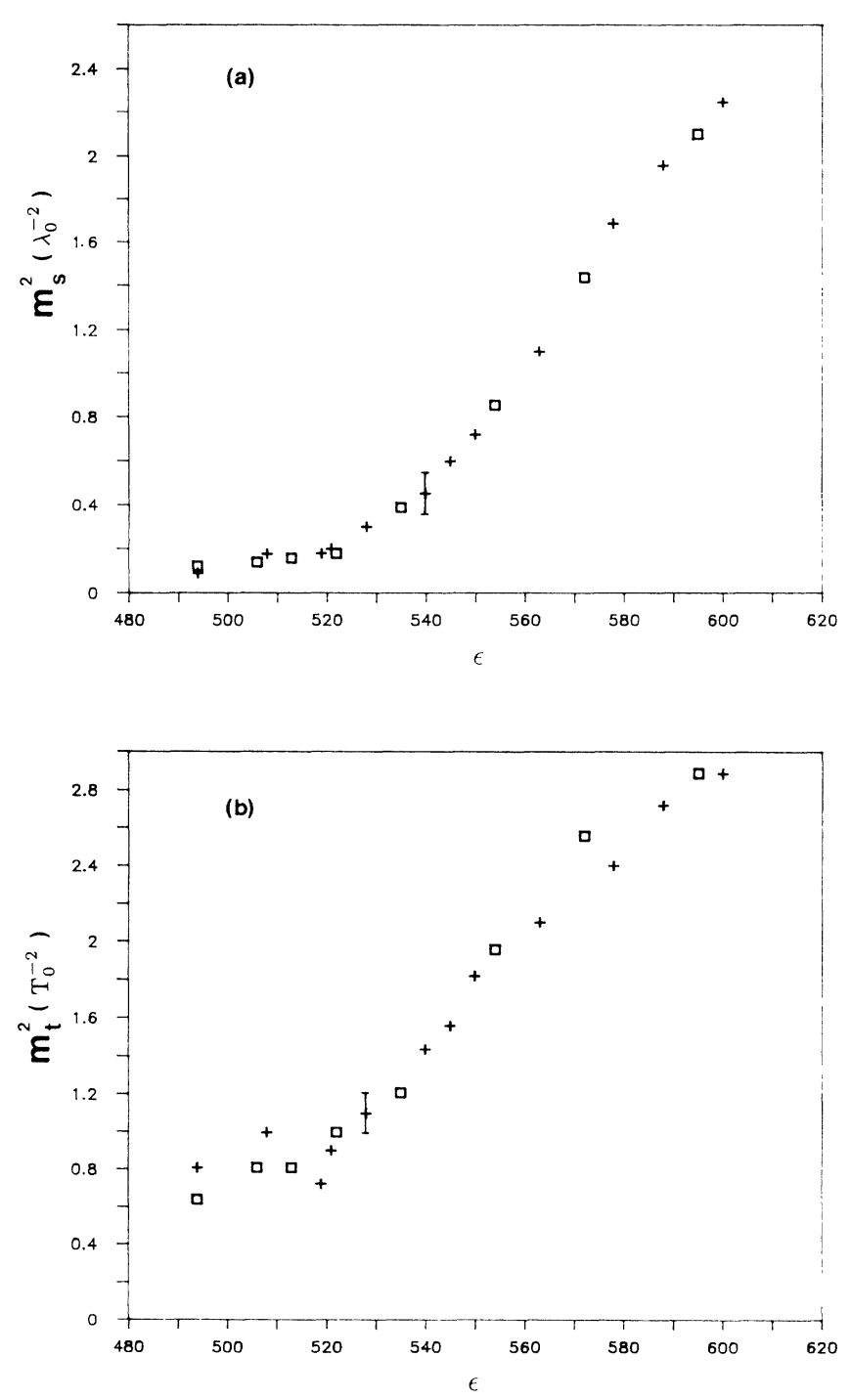

FIG. 9. Annular cell. (a) Square of the slope $m_{s}$ computed from the exponential decay of the histograms of the laminar domains, as a function of $\epsilon$. Crosses and rectangles correspond to increasing and decreasing $\epsilon$, respectively. (b) Square of the slope $m_{t}$ computed from the exponential decay of the histograms of the laminar domains with lifetime $\tau$ as a function of $\epsilon$.
The distributions of the lifetime of LD's have also been studied. By analyzing our space-time series with the same spatial criterion to discriminate laminar from turbulent cells, we have performed histograms giving the number $N(\tau)$ of laminar periods lasting $\tau$. This temporal approach displays features similar to those obtained in the spatial distributions. The histogram shows an algebraic decay for $\epsilon_{t}^{a}=490 \pm 10$ with a characteristic exponent $\mu_{t}^{a}=2 \pm 0.1$, and an exponential decay for $\epsilon>500$. The threshold of STI is indeed the same $\left(\epsilon_{s}^{a} \approx \epsilon_{t}^{a}\right)$ as for the spatial statistics even if the exponents are slightly different. In the case of an exponential decay, a characteristic time $l_{t}=1 / m_{t}$ is defined and $m_{t}^{2}$ varies also linearly with $\epsilon$ [Fig. 9(b)] if we skip the low values of $\epsilon$.

\section{B. STI in rectangular geometry}

We have performed the same spatiotemporal statistical analysis for the rectangular geometry. The results happen to be similar to those of the annular geometry, except for the fact that the transition - via STI - in this linear array of convective rolls is quasiperfect.

The evolution towards sustained STI has been first quantified via frequency-wave-number power spectra computed from the temporal evolution of the rolls of Fig. 4. Near the threshold of STI and for increasing $R$, a large broadening, in wave number, around the mean wave number is noticed as shown in Fig. 10(a). At larger $\mathcal{R}$, a broadening of the peaks takes place in the frequency domain. We conjecture that this is mainly due to an uncorrelated dynamics of the rolls and differs strongly from the time fluctuations of the oscillators. From these 2D Fourier transforms, no propagation of defects, such as the solitary waves observed near the threshold of STI, can be revealed. Such frequency-wave-number spectra also show that the transition toward sustained STI is at first initiated by a spatial destabilization of the uniform convective pattern giving a wavelength dispersion, followed, at higher $\mathcal{R}$, by time fluctuations of the new spatial pattern.

The distributions of spatial and temporal lengths of LD's exhibit qualitatively the same kind of behavior as in the annular cell. The histograms show a power-law decay near $\epsilon=\epsilon_{s}^{r}=360 \pm 10$, while they reveal an exponential decay for $\epsilon>380$ [Fig. 11(a)]. The characteristic length $l_{s}=1 / m_{s}$ and time $l_{t}=1 / m_{t}$ have a well-defined dependence on $\epsilon$, as

$$
\begin{aligned}
& m_{s} \sim\left(\epsilon-\epsilon_{s}^{r}\right)^{\alpha_{s}^{r}}, \quad \alpha_{s}^{r}=0.5 \pm 0.05, \\
& m_{t} \sim\left(\epsilon-\epsilon_{s}^{r}\right)^{\alpha_{t}^{r}}, \quad \alpha_{t}^{r}=0.5 \pm 0.05 .
\end{aligned}
$$

Probably due to finite-size effects (the container contains only 60 rolls), the power-law distributions are only defined on a small range of scales and therefore it is difficult to find a precise value of $\mu_{s}^{r}$ or $\mu_{t}^{r}$. An estimation gives $\mu_{s}^{r}=1.6 \pm 0.2$ and $\mu_{t}^{r}=2.0 \pm 0.2$. On the other hand, and contrary to the annular case, a unique threshold for STI is defined. In fact, the value of the threshold $\epsilon_{s}^{r}$ obtained from the distribution displaying a power law and the value $\epsilon_{F}^{r}$ defined from the law of evolution of $F_{t}$ on $\epsilon$ 

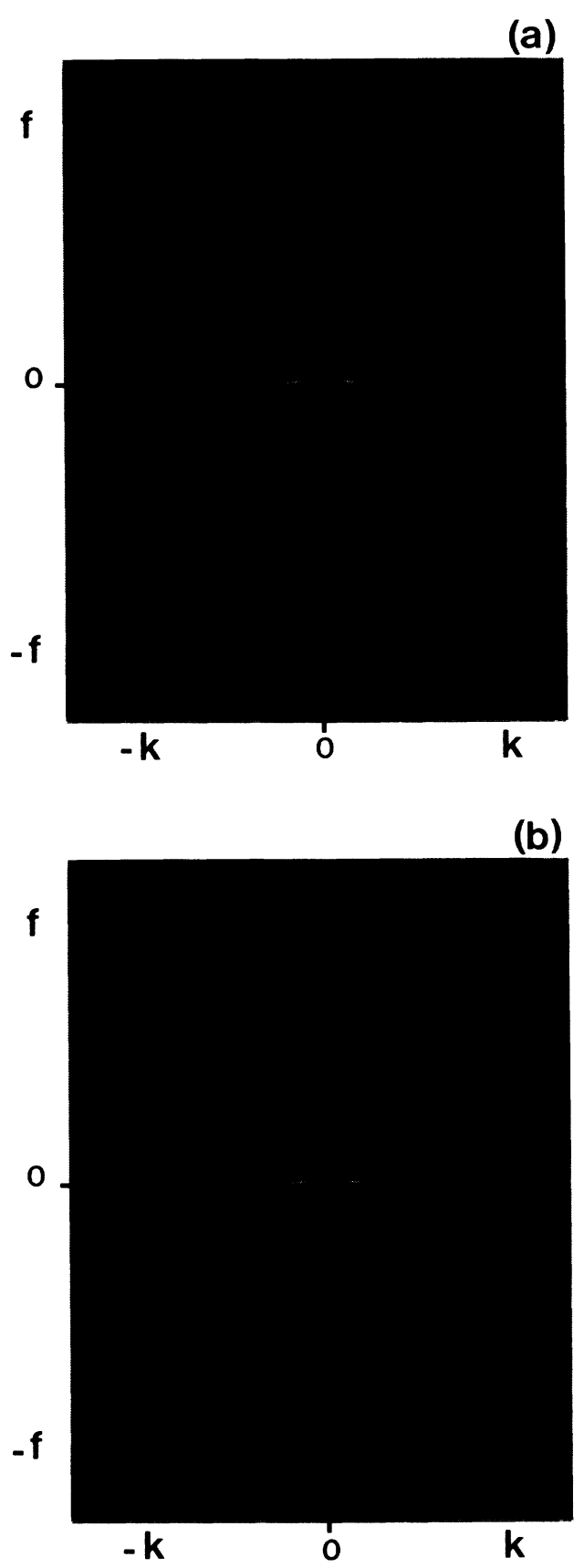

FIG. 10. 2D centered power spectra. Horizontal and vertical axis are wave-number and frequency axis, respectively. The maximum wave number and frequency are $1 / 8 \mathrm{~cm}^{-1}$ and $1 / 6$ $\mathrm{Hz}$. The logarithmic power spectra are plotted above a given threshold. (a) $\epsilon=380$, (b) $\epsilon=409$.

are the same, $\epsilon_{s}^{r}=\epsilon_{F}^{r} \simeq 360$. This value also corresponds to the first events of turbulence which can be observed in the experiment $\left(\epsilon^{r} \simeq 350\right)$. Accordingly, the transition via STI appears to be almost perfect in the rectangular container, but with exponents which do not differ within the error bars from these of the annular geometry.

To get more information on the transition process, we have also computed the correlation length $\xi$ of our sys-
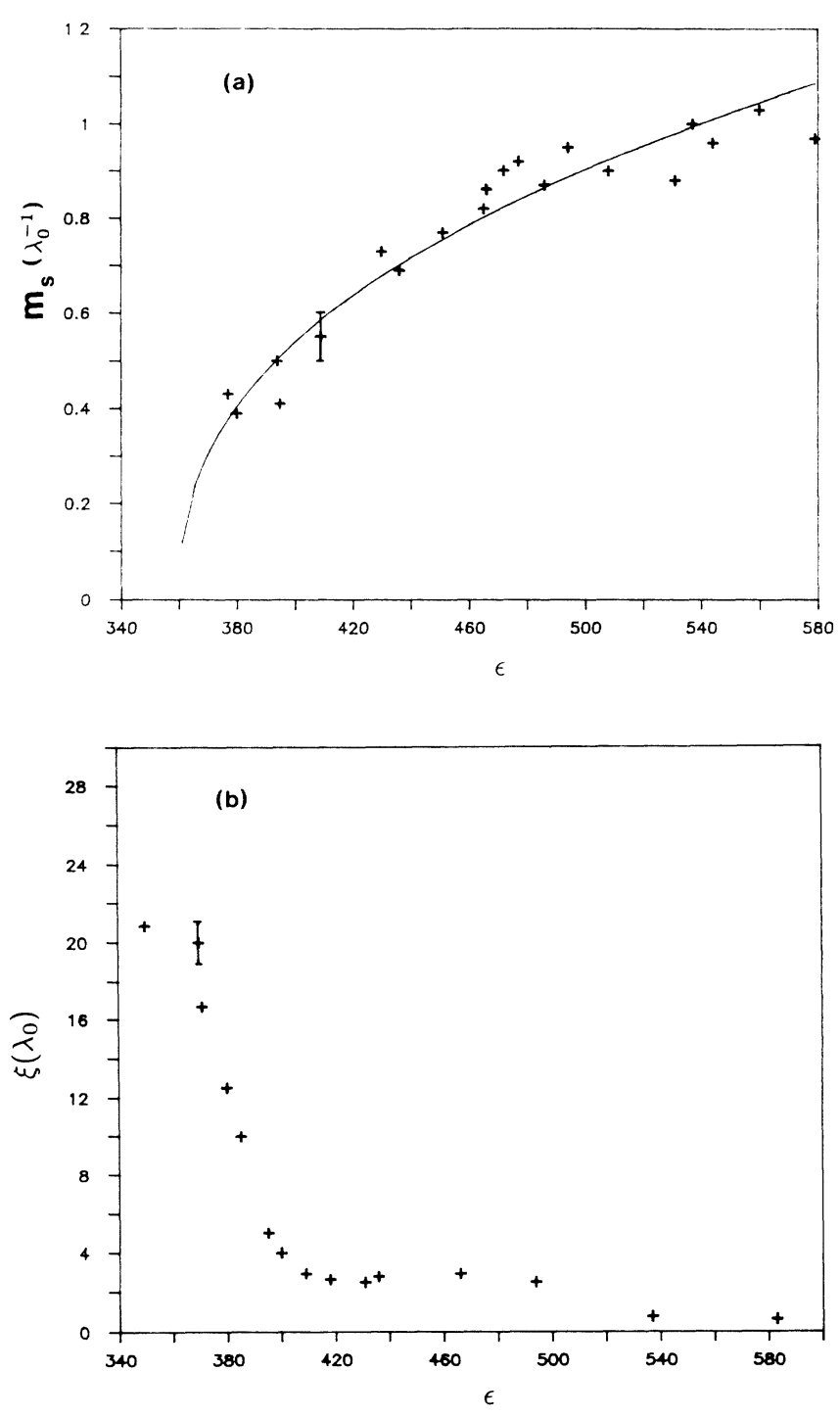

FIG. 11. Rectangular cell. (a) Slope $m_{s}$ computed from the exponential decay of the histograms of the laminar domains, as a function of $\epsilon$. (b) Spatial correlation length $\xi$ as a function of $\epsilon$. Notice the strong decrease of $\xi$ when the convective pattern enters into the spatiotemporal regime at $\epsilon=370$.

tem. $\xi$ is defined through the spatial correlation function:

$$
C(r)=\langle[I(r, t)-\langle I\rangle][I(0, t)-\langle I\rangle]\rangle /\left\langle I^{2}\right\rangle,
$$

where $I(r, t)$ and $I(0, t)$ are the light intensity at two points separated by a distance $r$ and \langle\rangle denotes the ensemble average. The envelope of the correlation function $C(r)$ is expected to decay as

$$
C(r) \sim \exp (-r / \xi),
$$

where $\xi$ is the correlation length to be compared to the length $L_{e}$ of the system. As stressed by Hohenberg and Shraiman, ${ }^{15}$ when $\xi>L_{e}$ which is the case for all small systems, the fluctuations may be regular or chaotic in time but are still coherent in space. On the other hand, 
for $\xi<L_{e}$, the dynamical behavior is incoherent in space and at moderate Rayleigh number is typical of a regime of weak turbulence without any energy cascade. This is the case for the convective regime studied in the two geometries.

Correlation functions of the intensity were computed to measure the correlation length $\xi$ for different values of $\epsilon$. The envelopes of these functions have an exponential decay with the distance $r$, from which $\xi$ can be extracted. Fig. 11(b) shows the dependence of $\xi$ on $\epsilon$ and reveals a change of behavior near $\epsilon=370$, where a sudden decrease of the correlation length is observed. Below the threshold of STI, the correlation length is of the order of the length of the system: $\xi \simeq 20 \lambda \sim L_{e}$, while far beyond $\epsilon_{s}^{r}$, $\xi \simeq \lambda<L_{e}$ which corresponds to the observed regime of spatiotemporal chaos.

A characteristic frequency was also computed from the first moment of the averaged frequency power spectra. ${ }^{16}$ A monotonic increase of the frequency is observed as a function of $\epsilon$; however, no saturation of the frequency value can be seen beyond the sustained STI regime.

\section{COMPARISON OF EXPERIMENTS WITH DIRECTED PERCOLATION SIMULATIONS}

The results of spatiotemporal analysis performed in the rectangular geometry are typical of a second-order phase transition, but the transition turns out to be imperfect in the case of the annular geometry. The nature and the characteristics of the transition will be discussed in Sec. VI. In this section we compare first the experimental measurements with available numerical results. Some statistical properties obtained with a very simple model constructed from direct percolation are then given.

First of all, the experiments reveal the same qualitative route to turbulence via STI as in the numerical simulations of some partial differential equations (PDE's), coupled map lattices, and directed percolation (DP) and most of the characteristic properties observed in the critical region around the threshold of STI are similar to those obtained numerically. The Kuramoto-Sivashinsky equation with an additional damping term reveals a second-order transition, with an algebraic decay close to the threshold, in the histograms giving the distribution of lengths of LD's, ${ }^{1}$ although the critical exponents are different from the experimental ones. A one-dimensional array of maps coupled by diffusion exhibits also the same behavior. In this last case, Chate and Manneville have shown that coupled map lattices display critical properties analogous to DP, but that they do not belong to the same universality class. ${ }^{5}$ The relationship between DP and the transition to turbulence via STI had been pointed out by Pomeau, ${ }^{9}$ who has placed the transition to turbulence for weakly confined systems in the field of critical phenomena.

To go further in this comparison between DP and experiments, we have performed calculations with a simple model of cellular automaton. In our problem, the automaton is defined on a 2D lattice: one space dimension made of $N$ sites (standing for the 1D geometry of our experiments) plus time. Each site has two possible states: a laminar state $(L)$ and a turbulent state $(T)$ by identification, respectively, with a laminar and a turbulent cell in the experiments. The probabilistic rules are defined on a three-site neighborhood, because the experiments suggest the influence of the two nearest convective rolls on the dynamical regime of the roll they surround. The state of a cell $i$ at time $t+1$ thus depends on its state and on the states of its neighbors $i-1$ and $i+1$ at time $t$.

In DP, the laminar state is absorbing, i.e., it cannot become turbulent if its parents are laminar: $p(L L L$ $\rightarrow T)=0$. However, since the experimental data show that a turbulent region may originate from a laminar one around the threshold of STI, a small probability $\widetilde{p}$ is introduced so that

$$
p(L L L \rightarrow T)=\widetilde{p} .
$$

This probability stands for the spontaneous apparition of turbulent spots in LD's.

The automaton is defined by a set of eight elementary probabilities $\left\{p_{k} \mid k=0,7\right\}$ :

$$
\begin{aligned}
& p_{0}=p(L L L \rightarrow T), \quad p_{1}=p(L L T \rightarrow T), \\
& p_{2}=p(L T L \rightarrow T), \quad p_{3}=p(L T T \rightarrow T), \\
& p_{4}=p(T L L \rightarrow T), \quad p_{5}=p(T L T \rightarrow T), \\
& p_{6}=p(T T L \rightarrow T), \quad p_{7}=p(T T T \rightarrow T) .
\end{aligned}
$$

The complementary probabilities are given by the relation $p(X Y Z \rightarrow L)=1-p(X Y Z \rightarrow T)$ and because of leftright symmetries, we have $p_{1}=p_{4}$ and $p_{3}=p_{6}$. We have chosen the case of directed bond percolation, which seems to be more representative of our experiments than site percolation, because of the diffusion process. Moreover, in order to make the calculations easier, we have taken totalistic rules, ${ }^{17}$ so that the following relations are satisfied:

$$
\begin{aligned}
& p_{1}=p_{2}=p_{4}=p, \\
& p_{3}=p_{5}=p_{6}=1-(1-p)^{2}, \\
& p_{7}=1-(1-p)^{3} .
\end{aligned}
$$

We are thus dealing with only one control parameter $p$ plus the probability $p_{0}=\tilde{p}$. For the numerical simulations, the number of sites $N$ was $10^{4}$ and the duration of the run was several $10^{4}$. The statistical analyses were performed only after some $10^{4}$ iterations to skip the transient regimes, especially near threshold. These values are not large enough to ensure accurate results of the exponents, which also depend on the size of the lattice. ${ }^{5}$ However, they allow a description of the main characteristics of the studied behaviors.

First of all, when $\widetilde{p}=0$, the results show a transition for $p=p_{c} \simeq 0.44$. The fraction of turbulent sites (the time average of the fraction of the system occupied by TD's) varies as

$$
F_{t} \sim\left(p-p_{c}\right)^{\beta}
$$

with $\beta \simeq 0.28$ and, for $p=p_{c}$, the histograms giving the distribution of LD's of length $L$ show a power-law decay 
with a characteristic exponent $\mu_{s}(\widetilde{p}=0) \simeq 1.5$. Moreover, for $p>p_{c}$, the histograms exhibit an exponential decay with a characteristic length $l_{s}=1 / m_{s}$ which depends on $p$. The evolution of $m_{s}$ can be fitted by

$$
m_{s} \sim\left(p-p_{c}\right)^{\alpha_{s}}, \quad \alpha_{s} \simeq 1
$$

The distributions of the lifetime of LD's evidence the same transition at $p=p_{c}$ and give an exponent $\mu_{t}(\widetilde{p}=0) \simeq 1.6$ and a characteristic time $l_{t}=1 / m_{t}$ for $p>p_{c} \cdot m_{t}$ depends on $p$ as

$$
m_{t} \sim\left(p-p_{c}\right)^{\alpha_{t}}, \quad \alpha_{t} \simeq 1 \text {. }
$$

A correlation length $\xi$ can also be defined from the spatial correlation function which decays as $C(r)$ $\sim \exp (-r / \xi)$. The variation of $\xi$ reveals a transition near $p=p_{c}$ which varies as

$$
\xi \sim\left(p-p_{c}\right)^{v}
$$

with $v_{s} \simeq 0.5$. A second-order phase transition is therefore observed when $\tilde{p}=0$, with well-defined threshold and characteristic exponents.

The results obtained when $\tilde{p} \neq 0$ display several important differences from the case $\tilde{p}=0$. First of all, the spatiotemporal representations of the automaton exhibit features very similar to the experimental results. It shows the importance of the spontaneous appearance of turbulent sites for the generation of localized TD's near the threshold $p_{c}$, while for $p \gg p_{c}$, the role of $\widetilde{p}$ is totally hidden by the process of contamination of LD's by TD's.

The statistical properties are also changed by the presence of $\widetilde{p}$. If it is small enough $\left(\widetilde{p}<10^{-3}\right)$, an algebraic decay is still observed in the histograms giving the distribution of LD's, but in a range of length scales which shortens when $\widetilde{p}$ increases. The characteristic exponent also depends on $\widetilde{p}$. With $\widetilde{p}=5 \times 10^{-4}$, the histograms show an algebraic decay around $p=0.44$ on 100 sites with a characteristic exponent $\mu_{s}\left(\tilde{p}=5 \times 10^{-4}\right) \simeq 1.4$. On the other hand, no noticeable change is observed in the distributions of lifetime of LD's, provided $\widetilde{p}$ is small enough: $\mu_{t}(\tilde{p}=0) \simeq \mu_{t}\left(\tilde{p}=5 \times 10^{-4}\right)$. When $\tilde{p}$ is increased further, no transition in the functional form of the distributions of LD's is observed: all the histograms show an exponential decay. This last result is similar to the conclusions of Bagnoli et al. ${ }^{18}$ who have also compared the behavior of the experimental data of Ciliberto and Bigazzi $^{7}$ with that of suitable chosen automaton rules.

When $\tilde{p} \neq 0$, a change of behavior of the turbulent fraction is still observable (Fig. 12). The value of $F_{t}$ for $p=p_{c}$ is different from zero and increases with $\widetilde{p}$. In fact, if $\tilde{p}>10^{-3}, F_{t}$ evolves slowly with $p$ and reveals no transition. The evolution of $m_{s}$ (or $m_{t}$ ) with $p$ is also affected by an increase of $\widetilde{p}$, as the evolution of $F_{t}$.

In conclusion, these numerical results show the existence of a well-defined transition in the case of directed percolation, i.e., when the laminar state is absorbing $(\widetilde{p}=0)$. When the laminar state is quasiabsorbing $(\widetilde{p} \neq 0)$, the transition disappears in a strict sense or becomes imperfect. The evolution of the turbulent fraction becomes

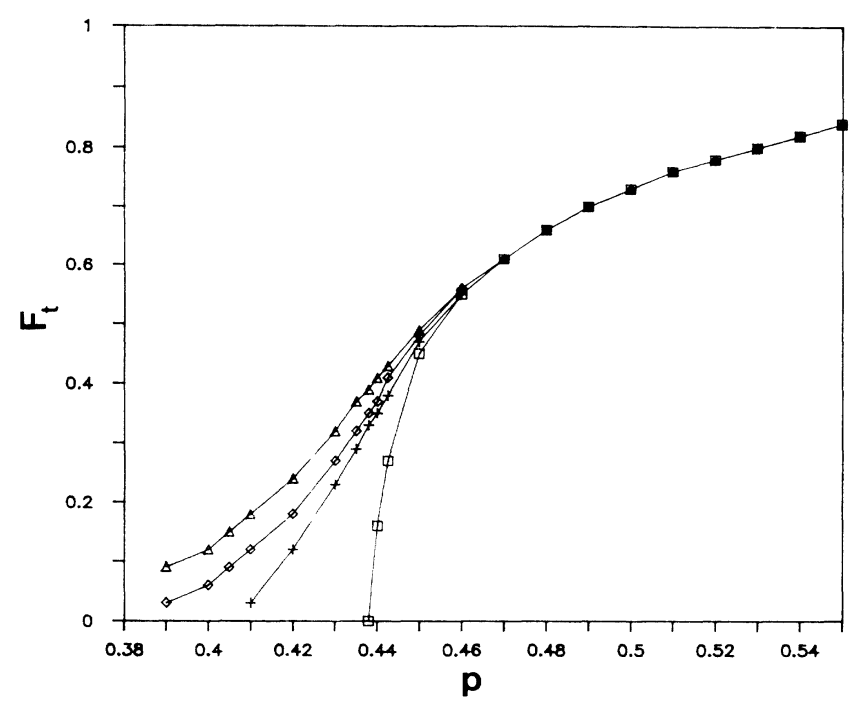

FIG. 12. Mean turbulent fraction $F_{t}$ as a function of the probability $p$ for different $\widetilde{p}$ (see text). Squares, $\widetilde{p}=0$; crosses, $\widetilde{p}=5 \times 10^{-4} ;$ diamonds, $\widetilde{p}=10^{-3} ;$ triangles, $\widetilde{p}=2 \times 10^{-3}$.

smooth; meanwhile, the algebraic decay, which is characteristic of the critical transition, disappears when $\widetilde{p}$ increases.

Finally, these numerical results performed on a very simple model of DP give an interesting insight on the experimental data. The rectangular geometry is well represented by pure DP; meanwhile, the annular case can be compared to DP with $\widetilde{p} \neq 0$. Though the physical nature of $\widetilde{p}$ is not explained, it gives a good representation of the spontaneous apparition of turbulent cells. As a power-law decay is present in the experimental distributions, it turns out that the probability $\widetilde{p}$ is small in the experiments, as shown by the numerical results. The laminar state of the convective rolls is thus quasiabsorbing.

\section{DISCUSSION}

The experimental results discussed in this paper have shown that in quasi-1D Rayleigh-Benard convection, the evolution of the convective state to sustained spatial turbulence is achieved through the development of spatiotemporal intermittency, as expected from theoretical and numerical studies. The observation and quantitative analysis of STI regimes have been previously performed in convection experiments, ${ }^{7}$ but in that case, the $1 \mathrm{D}$ character was not well fulfilled, and the criterion to separate LD's from TD's was different from that used in the present study. Nevertheless, many properties remain similar in the two cases, showing the robustness of the STI regimes.

In the present study, two different geometries were used. Periodic boundary conditions were obtained using an annular cell, while fixed boundary conditions prevailed in the rectangular channel. The transverse aspect ratio was slightly different for the two cases, but this difference seemed to influence only the dynamical regimes preced- 
ing STI. As a matter of fact, the first time-dependent convective behavior was given by the appearance of oscillators in the convective pattern - plumes in the rectangular channel, vacillations in the annulus -in agreement with the general observation that vacillations do not exist when $\Gamma_{y}>0.4$. Nevertheless, the qualitative behavior of the sustained STI regime was exactly the same in both geometries, therefore it is independent of the nature of the oscillators in the convective pattern. Their influence on the STI threshold through eventual specific mechanisms of spatial destabilization is unknown, but it is likely that the observed difference $\left(\epsilon^{r}=360, \epsilon^{a}=480\right)$ is related only to the value of $\Gamma_{y}$ itself (in the experiment reported in Ref. 7, the threshold value $\epsilon^{a}=200$ with $\Gamma_{y}=1$ ).

In the two geometries, the spatiotemporal intermittent regime corresponds to a loss of spatial coherence and appears as an interspersing of turbulent patches within laminar domains. Very close to the STI threshold, turbulent patches spontaneously appear from time to time and, after a while, relax and disappear. The intermittent behavior of a complete ordered pattern with a locally turbulent one is observed within a very narrow $\epsilon$ interval in the rectangular channel, and within a larger $\epsilon$ domain in the annulus, as is clearly evidenced by the variation of the turbulent fraction as a function of $\epsilon$. Moreover, this variation shows that in the former case, we have a quasiperfect second-order phase transition. In the case of the annulus, the nature of the transition from laminar state to STI is more complex and looks imperfect, though the statistics concerning the length of the laminar domains (power law at the "effective" threshold, scaling law for the characteristic length) are similar to the statistics expected for a well-defined transition. A somewhat similar behavior has been reported in Ref. 7, where the convection is also built up in an annular container (with $\Gamma_{y}=1$ ). The striking difference between the behaviors in the annular and rectangular geometries is probably due to the influence of the boundaries which stabilizes the phase of the rolls in the rectangular channel. On the contrary, in the annular geometry, an intrinsic phase roll instability together with a possible large scale flow could induce the observed imperfect transition. In fact, it is only in this geometry that turbulent patches propagate near the threshold.

The experimental results agree globally with those obtained by numerical simulation of PDE or coupled maps and also with directed percolation. The quantitative comparison between the values of the exponents numerically and experimentally measured (see Table I) is not straightforward, for recent studies ${ }^{5,19}$ show that the exponents are not universal. They depend, in particular, on the size of the system, but also on the underlying mechanisms which govern the transition. Nevertheless, the exponents $\beta^{r}$ - for the evolution of the turbulent fraction in the rectangular geometry - and $\mu_{s}$ - of the algebraic decay-deduced from the experimental observations, may agree with those of DP and coupled maps. To our knowledge, the prediction concerning the $\alpha$ exponent describing the evolution of the inverse of the characteristic length as a function of $\epsilon$, has not yet been done. The exponent obtained through our numerical simulation of directed percolation is not the same as from the experimental data. Furthermore, no clear correspondence between the two control parameters $p$ and $\epsilon$ can be established. A recent phenomenological study of a 1D chain of rolls, ${ }^{20}$ performed in the frame of the LandauGinsburg equation, ${ }^{21}$ could give a transition similar to our experimental observations.

The recent studies of $1 \mathrm{D}$ periodic systems have revealed the richness of their spatial and dynamical properties. In a certain range of the control parameter, they present an analogy with a chain of coupled oscillators, and so, are linked to dynamical systems. Then, the first symmetry breaking of the space translation takes place by the appearance of propagative solitary waves. These waves, observed in the convective narrow channels, have been also evidenced in directional solidification ${ }^{22,23}$ and in the periodic front of the meniscus of a fluid between two rotating cylinders. ${ }^{24}$ In this last experiment, spatiotemporal intermittency is also currently under investigation. Therefore it seems that a large amount of the properties we have found is generic to $1 \mathrm{D}$ systems.

TABLE I. Values of different exponents

\begin{tabular}{|c|c|c|c|c|c|}
\hline \multicolumn{6}{|c|}{ Experimental results } \\
\hline Geometry & $\beta$ & $\mu_{s}$ & $\mu_{t}$ & $\alpha_{s}$ & $\alpha_{t}$ \\
\hline $\begin{array}{l}\text { Rectangular channel } \\
\text { Annulus } \\
\text { Annulus (Ref. 7) }\end{array}$ & 0.3 & $\begin{array}{l}1.6 \pm 0.2 \\
1.7 \pm 0.1 \\
1.9 \pm 0.1\end{array}$ & $\begin{array}{c}2 \pm 0.2 \\
2 \pm 0.1 \\
1.9\end{array}$ & $\begin{array}{l}0.5 \pm 0.05 \\
\simeq 0.5 \\
0.5\end{array}$ & $\begin{array}{c}0.5 \pm 0.05 \\
\simeq 0.5\end{array}$ \\
\hline \multicolumn{6}{|c|}{ Numerical results } \\
\hline $\begin{array}{l}\text { DP (this work) } \\
\text { (three parents) }\end{array}$ & $\simeq 0.28$ & 1.5 & 1.6 & $\simeq 1$ & $\simeq 1$ \\
\hline $\begin{array}{l}\text { DP (Ref. 5) } \\
\text { (two parents) }\end{array}$ & 0.28 & 1.6 & & & \\
\hline Coupled maps (Ref. 5) & $\simeq 0.25$ & $1.78,2$ & & & \\
\hline PDE (Ref. 1) & & 3.15 & & & \\
\hline
\end{tabular}




\section{ACKNOWLEDGMENTS}

We wish to acknowledge G. Balzer, P. Bergé, R. Bidaux, Y. Pomeau, and P. Manneville for fruitful and stimulating discussions. We would like to thank also $\mathbf{P}$. Hede for the development of the image processing and B. Ozenda for his technical assistance.
${ }^{1}$ H. Chaté and P. Manneville, Phys. Rev. Lett. 58, 112 (1987). According to a private communication with one of these authors, P. Manneville, the knowledge of the exact nature of the transition, studied in this paper, requires more studies.

${ }^{2}$ U. Frisch, Z. S. She, and O. Thual, J. Fluid Mech. 168, 221 (1986).

${ }^{3}$ B. Nicolaenko, Nucl. Phys. B (Proc. Suppl.) 2, 453 (1987); H. Chaté and B. Nicolaenko, in New Trends in Non-Linear Dynamics and Pattern Forming Phenomena: the Geometry of Nonequilibrium, edited by P. Huerre and P. Coullet (Plenum, New York, 1990).

${ }^{4}$ K. Kaneko, Prog. Theor. Phys. 74, 1033 (1985).

${ }^{5}$ H. Chaté and P. Manneville, Physica D 32, 409 (1988); J. Stat. Phys. 56, 357 (1987).

${ }^{6}$ P. Bergé, Nucl. Phys. B 2, 247 (1987).

${ }^{7}$ S. Ciliberto and P. Bigazzi, Phys. Rev. Lett. 60, 286 (1988).

${ }^{8}$ F. Daviaud, M. Dubois, and P. Bergé, Europhys. Lett. 9, 441 (1989).

${ }^{9}$ Y. Pomeau, Physica D 23, 3 (1986).

${ }^{10}$ M. Dubois, P. Bergé, and A. Petrov, in New Trades in NonLinear Dynamics and Pattern Forming Phenomena: the Geometry of Nonequilibrium, edited by P. Huerre and P. Coullet (Plenum, New York, 1990).

${ }^{11}$ J. E. Wesfreid and V. Croquette, Phys. Rev. Lett. 45, 634 (1980); V. Croquette and F. Schosseler, J. Phys. 43, 1183 (1982).

${ }^{12}$ M. Dubois, R. Da Silva, F. Daviaud, P. Bergé, and A. Petrov,
Europhys. Lett. 8, 135 (1989).

${ }^{13}$ M. Dubois, F. Daviaud, and M. Bonetti, in Non-Linear Evolution of Spatio-Temporal Structures in Dissipative Continuous Systems, edited by F. H. Busse and K. Kramer (Plenum, New York, 1989).

${ }^{14}$ H. Chaté, Ph.D. thesis, University of Paris, 1989.

${ }^{15}$ P. C. Hohenberg and B. I. Shraiman, Physica D 37, 109 (1989).

16J. P. Gollub and R. Ramshankar, New Perspectives in Turbulence, edited by S. Orszag and L. Sirovich (Springer-Verlag, Berlin, in press).

${ }^{17}$ W. Kinzel, Ann. Israel Phys. Soc. 5, 425 (1983).

${ }^{18}$ F. Bagnoli, S. Ciliberto, A. Francescato, R. Livi, and S. Ruffo, in Chaos and Complexity, edited by R. Livi, S. Ruffo, S. Ciliberto, and M. Buiatti (World Scientific, Singapore, 1988).

${ }^{19}$ R. Bidaux, N. Boccara, and H. Chaté, Phys. Rev. A 39, 394 (1989).

${ }^{20}$ P. Coullet and G. Iooss, Phys. Rev. Lett. 64, 866 (1990).

${ }^{21}$ J. Lega and P. Coullet (private communication).

${ }^{22}$ A. J. Simon, J. Bechhoefer, and A. Libchaber, Phys. Rev. Lett. 61, 2574 (1988); P. Coullet, R. E. Goldstein, and G. H. Gunaratne, ibid. 63, 1954 (1989).

${ }^{23}$ G. Faivre, S. de Cheveigné, C. Guthmann, and P. Kurowski, Europhys. Lett. 9, 779 (1989).

${ }^{24}$ M. Rabaud, S. Michalland, and Y. Couder, Phys. Rev. Lett. 64, 184 (1990). 


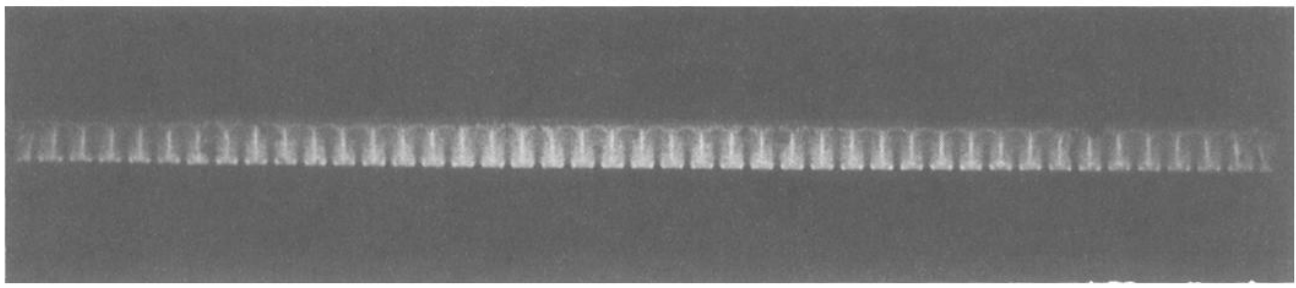

FIG. 1. Shadowgraphic image of 1D Rayleigh-Bénard convection in a rectangular container with $\Gamma_{y}=0.3$ at $\epsilon=45$. Bright and dark lines are cold and warm currents, respectively. 
(a)

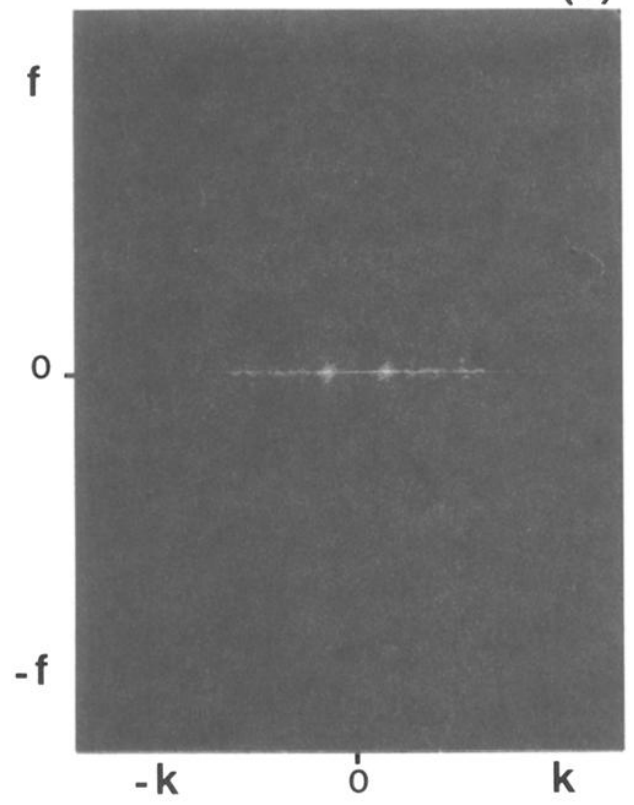

(b)

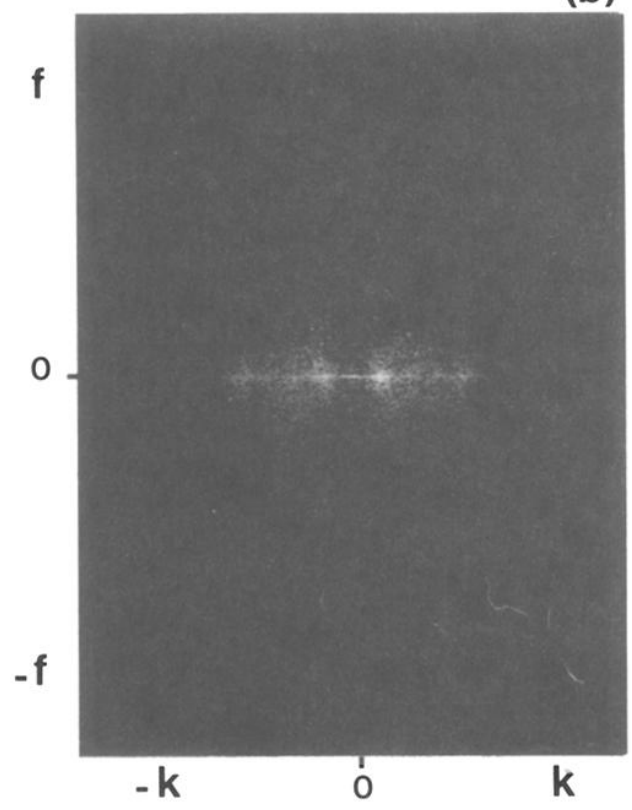

FIG. 10. 2D centered power spectra. Horizontal and vertical axis are wave-number and frequency axis, respectively. The maximum wave number and frequency are $1 / 8 \mathrm{~cm}^{-1}$ and $1 / 6$ Hz. The logarithmic power spectra are plotted above a given threshold. (a) $\epsilon=380$, (b) $\epsilon=409$. 


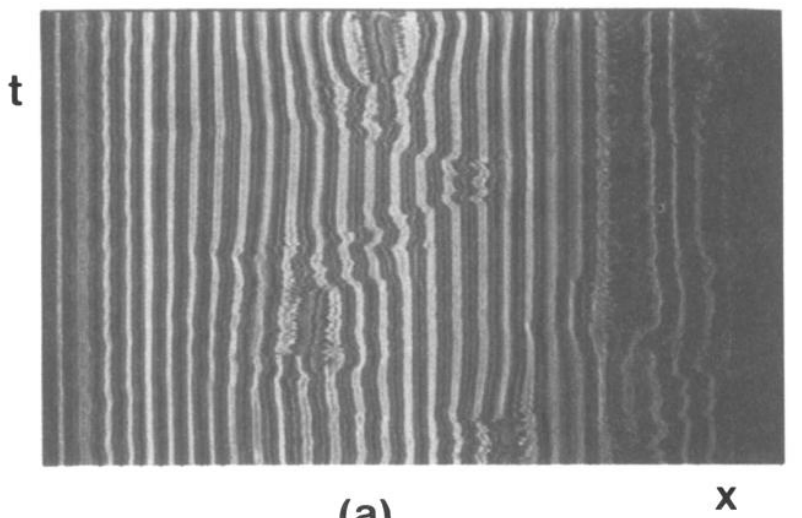

(a)
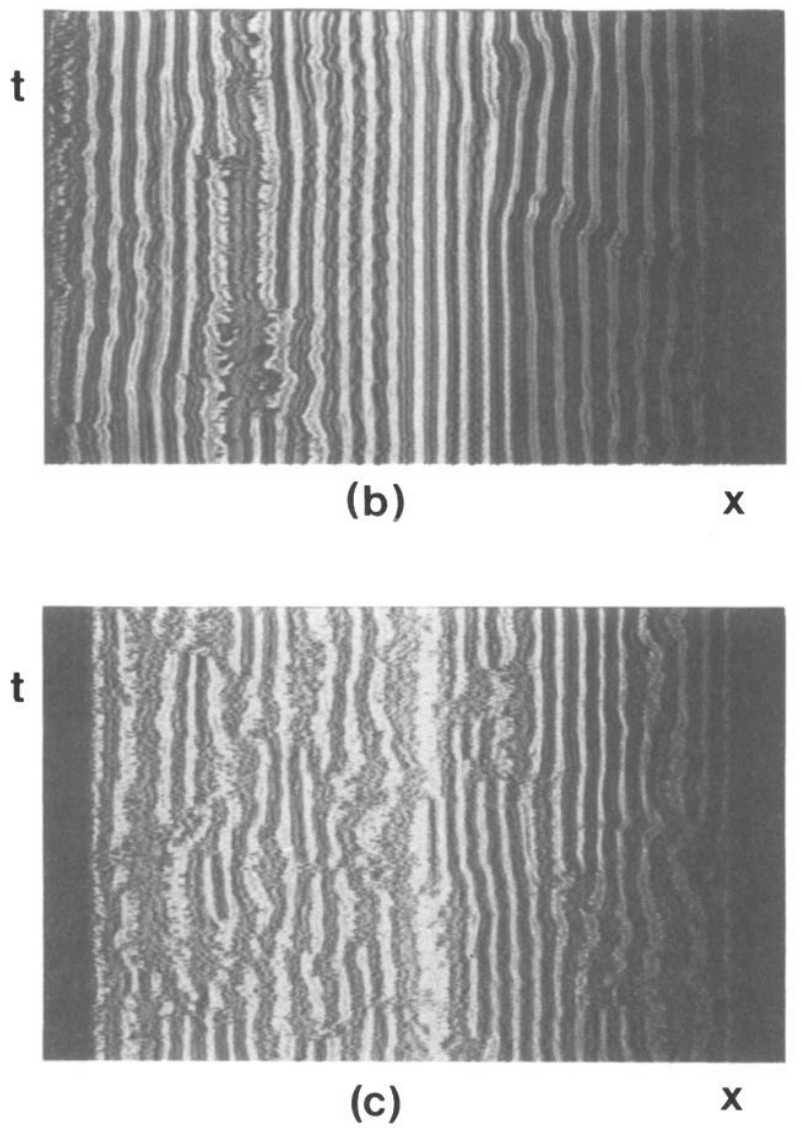

FIG. 4. Space-time evolution of the convective pattern obtained in the rectangular channel from the shadowgraphic images. The light intensity is plotted using a gray scale with 256 levels. Spatial digitization is made over 512 pixels (horizontal axis). The total observation time (vertical axis) is $1536 \mathrm{sec}$. Vertical dark lines correspond to stationary warm ascending currents. (a) $\epsilon=350$, (b) $\epsilon=409$, (c) $\epsilon=561$. 

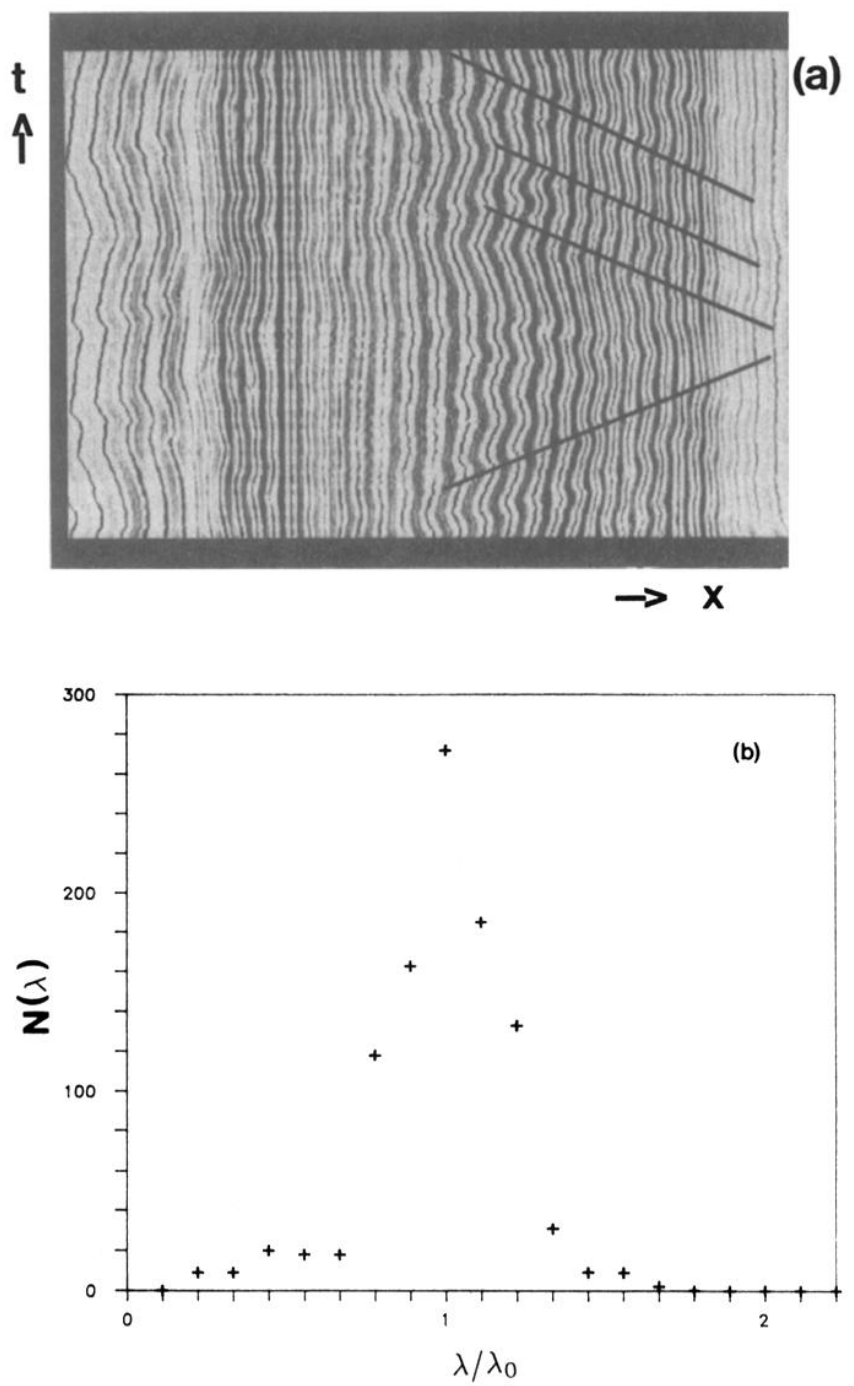

FIG. 5. (a) Same as in Fig. 4 but with the annular channel. The total observation time is $1500 \mathrm{sec}$ and $\epsilon=420$. Notice at the right and left of the frame the crossing of solitary waves. Dark lines indicate the trajectory of some solitary waves. (b) Wavelength distribution at $\epsilon=420$. 


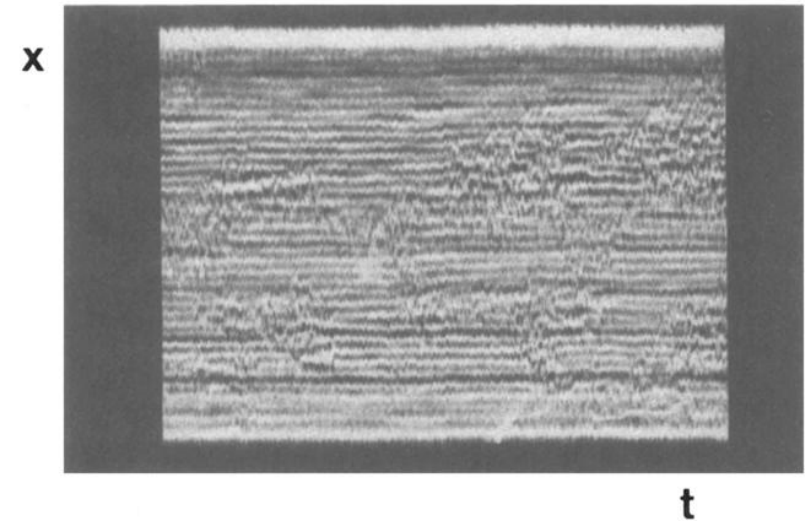

FIG. 6. Same as in Fig. 4 but with the annular convective pattern at $\epsilon=520$. The total observation time is $1000 \mathrm{sec}$ (along the horizontal axis, time is going from left to right) and the spatial digitization is made over 250 pixels. Notice the contamination process of the turbulent domains throughout the laminar ones. 